\title{
Break on through to the other side: outer membrane penetration of the nascent flagellum by a stop-polymerization mechanism
}

\author{
Edgar Huitema and Patrick H. Viollier ${ }^{1}$ \\ Department of Molecular Biology and Microbiology, School of Medicine, Case Western Reserve University, \\ Cleveland, Ohio 44106, USA
}

Bacterial cells are equipped with a myriad of organelles or complex protein assemblages that execute specialized functions required for cellular integrity and survival in the ever-changing environment. Of these organelles, the flagellum is renowned for its use as a model system to elucidate the intricate molecular mechanisms that direct and coordinate the precise subcellular placement and step-wise assembly of macromolecular structures (Pandza et al. 2000; Aldridge and Hughes 2002; Macnab 2003; Huitema et al. 2006; Lam et al. 2006; Murray and Kazmierczak 2006). Interrogating the latter process, Chevance et al. (2007) discovered a new conceptual mechanism that underlies flagellar self-assembly and possibly that of other complex protein assemblages.

\section{Flagellar mobility drives nanomachine research}

The flagellum, a sophisticated nanomachine embedded in the cell envelope, generates the thrust that propels cells in liquid or semisolid medium through rotation of an extracellular propeller-like structure (Macnab 1999). Flagellum-mediated motility formed the basis of robust phenotypic assays that unearthed the complete inventory of protein constituents that build the flagellum. Subsequent ultrastructural analyses showed that these constituents assemble into specialized flagellar substructures that are mechanically analogous to motor components of man-made machines (Fig. 1B; Macnab 1999). A basal body substructure embedded in the cell envelope encases a rotary motor, which is energized through the proton motive force, and a driveshaft substructure (the rod) (Murphy et al. 2006). The rod relays the power from the motor to a universal joint (the hook) that connects the basal body with the external propeller (the flagellar filament). Bacteria assemble the flagellum

${ }^{1}$ Corresponding author.

E-MAIL patrick.viollier@case.edu; FAX (216) 368-1055.

Article is online at http://www.genesdev.org/cgi/doi/10.1101/gad.1600807. with remarkable structural consistency, which has led many researchers to predict the existence of a sophisticated and inherent assembly program relying, at least in part, on self-assembly mechanisms.

Flagellum biogenesis nucleates on a protein platform (the MS-ring) in the cytoplasmic membrane, with construction subsequently extending outward through the cell envelope (Macnab 1999). The subunits are synthesized within the cell and delivered to the construction site, where they are incorporated into the various substructures. A flagellum-specific secretion apparatus transports these subunits at the expense of ATP hydrolysis to the distal tip of the nascent structure through a hollow conduit located in its center. Not surprisingly, the secretion apparatus is one of the earliest substructures built as part of the basal body. Of note, the secretion machinery functions as a gatekeeper that determines when the subunits for new substructures are exported, and thus, when these substructures can assemble (Minamino and Macnab 1999; Minamino et al. 1999; Aldridge and Hughes 2002). Since timely presentation of the substrates is critical, secretion must reflect the developmental status of the elongating structure. It is therefore likely that sensing and signal processing mechanisms exist to allow communication between the nascent structure and the secretory apparatus. In addition, elongation of the flagellar structure must be tightly coordinated with puncturing of envelope barriers. This process is particularly pertinent to Gram-negative bacteria, such as Salmonella enterica, where the basal body is embedded within three envelope layers: (1) a cytoplasmic membrane made up of a phospholipid bilayer, (2) a rigid cell wall stabilized through peptidoglycan crosslinks, and (3) an outer membrane comprising an inner leaflet of phospholipids and an outer leaflet of lipopolysaccharide (LPS). A substructure, known as the distal flagellar rod, protrudes through the cell wall and outer membrane via rings anchored in the peptidoglycan (Pring) and the LPS (L-ring) layer, respectively (Fig. 1B). In this issue of Genes \& Development, Chevance et al. 
A

Figure 1. Role of stop-polymerization in flagellum assembly processes. (A) Polymerization of FlgG into two monomeric stacks results in a conformation change (from $\alpha$ to $\left.\alpha^{\prime}\right)$. The resulting intermolecular rearrangement prevents further addition of FlgG monomers, thereby halting rod elongation (stop-polymerization [SP]). Stop-polymerization allows further stepwise assembly of the flagellum by addition of the P-ring (FlgI), L-ring, hook, and flagellar filament. $(B)$ The completed flagellum traverses the inner membrane (IM), the peptidoglycan layer (PG), and the outer membrane (OM) comprising lipopolysaccharide (LPS) in the outer leaflet. (C) Mutations in FlgG affect the conformational switch, abolishing the stop-polymerization signal and causing rod elongation. In this scenario, ectopic P-rings can form either due to absence of the stop-polymerization signal or through local conformational alterations that favor P-ring nucleation and that occur aberrantly at discrete sites along the elongated rod. $(D)$ Absence of the stop-polymerization signal affects outer membrane and perhaps peptidoglycan layer penetration and substrate preswitching of the Type III secretion system (T3SS). This results in formation of a periplasmic flagellum in FlgG point mutants. Finally, mutations in FlgG and rod elongation affect the timing of substrate secretion. FlgM, a negative regulator of "late" gene transcription, is prematurely secreted into the periplasm.

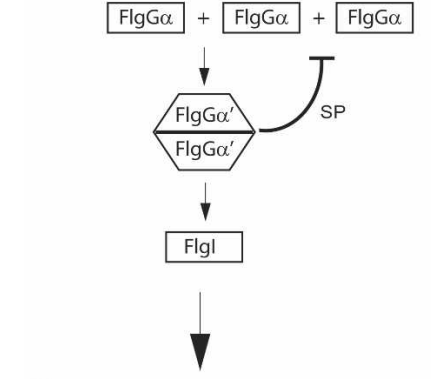

B

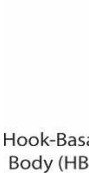

Body (HBB) Complex

.

C

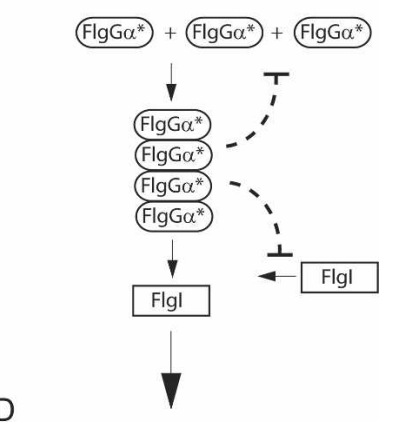

(2007) make important strides toward defining the elusive mechanism of outer membrane penetration by the distal rod. They report a class of mutations in the FlgG distal rod subunit that result in elongated rods that are unable to pierce the outer membrane (Fig. 1C,D). Based on the "runaway" polymerization phenotype of FlgG mutant rods, a crucial "stop-polymerization" mechanism is invoked that determines whether flagella puncture and traverse the outer membrane or whether they are confined to the periplasmic (intramembrane) space (Fig. 1A,C,D), as is the case for the flagella in spirochetes (Bardy et al. 2003). An important implication of this result is that inherent "stop-polymerization" mechanisms might not only underlie ordered "self-assembly" processes of flagella and structurally related organelles, but find prevalence in assembly processes of divergent proteinaceous structures.

\section{Flagellum assembly: reconciling sense with availability}

Over a decade ago, Hughes et al. (1993) already illuminated an enigmatic mechanism that helped explain how formation of one flagellar substructure is "sensed" and seamlessly linked to initiation of the next crucial event in flagellum biogenesis. Completion of the hook-basal body substructure (HBB) is accompanied by a switch in the secretion specificity from the subunits of early flagellar structures, such as the rod subunits, to a later subset that includes the FlgM regulator and the flagellins that are assembled into the filament (Fig. 1B; Karlinsey et al. 2000). FlgM is an inhibitor of $\sigma^{28}$-dependent tran- scription of flagellin genes (Gillen and Hughes 1991). The aforementioned substrate specificity switch is part of an important checkpoint mechanism that prevents premature flagellin synthesis. While the HBB is being built or when it is prevented from forming by mutation, FlgM is retained in the cytoplasm and flagellin transcription is inhibited. With the formation of the HBB and the resulting secretion switch, FlgM is exported into the supernatant (Karlinsey et al. 2000). This event drains the inhibitor from the cytoplasm, which permits expression of the flagellins. While this work outlined a logical framework for the process of coupling the assembly of one substructure to the next, the structural basis for the secretion switch was poorly understood. In an attempt to crack the molecular code of the switch, Chevance et al. (2007) used an elegant genetic screen to identify mutations that bypass the FlgM secretion block exhibited by strains that lack the $\mathrm{P}$ - and L-ring proteins and, thus, cannot complete the HBB (Aldridge et al. 2006; Chevance et al. 2007). In this situation, flagellin transcription can only be relieved by mutations that inactivate FlgM or that "rewire" the regulatory circuitry of FlgM secretion. It is the latter class of mutations that Chevance et al. (2007) sought to isolate using a $\sigma^{28}$-dependent reporter construct. A clever secondary screen was subsequently used to discriminate between FlgM loss-of-function mutations and those that bypass the FlgM secretion block. When the latter mutants bear an additional mutation in the flagellar secretion machinery that renders its function temperature sensitive, FlgM export is conditional. At the permissive temperature FlgM 
is exported and $\sigma^{28}$-dependent transcription can take place, while at the restrictive condition, FlgM export and flagellin transcription is prevented. In contrast, in strains with a FlgM loss-of-function mutation, $\sigma^{28}$-dependent transcription occurs either at the restrictive or at the permissive temperature. This strategy combined with cotransduction experiments to avoid reisolating previously characterized Flk mutants (Aldridge et al. 2006), led to the discovery of novel mutations in FlgG (henceforth referred to as $\mathrm{FlgG}^{\star}$ ) (Chevance et al. 2007). These $\mathrm{FlgG}^{\star}$ mutations allow FlgM drainage in the absence of $\mathrm{P}$ - and L-ring proteins, indicating that mutations in the rod can deregulate the $\mathrm{HBB}$ checkpoint.

\section{Stop-polymerization: putting the breaks on hot rods}

Since mutations in a structural component of the rod were not expected to impact regulation of FlgM secretion, Chevance et al. (2007) performed transmission electron microscopic analysis (TEM) of purified HBB structures in an attempt to elucidate the molecular effects of the FlgG ${ }^{\star}$ mutations. These studies revealed FlgG ${ }^{\star}$ distal rods to be considerably elongated compared with wild-type rods, suggesting a "stop-polymerization" defect that results in "runaway" polymerization of FlgG* subunits (Fig. 1C). In addition to their extended length, $\mathrm{FlgG}^{\star}$ rods also bore multiple P-rings, yet lacked L-rings. TEM revealed that FlgG $^{\star}$ mutant cells also lacked flagellar surface structures, but when cells were plasmolyzed by osmotic shock treatment, flagella were observed, implying that they reside in the periplasmic space of $\mathrm{FlgG}^{\star}$ mutants. Thus, $\mathrm{FlgG}^{\star}$ mutations recode the assembly program to produce periplasmic flagella rather than surface exposed ones (Fig. 1D). A likely basis for this effect is the assembly of FlgG $^{\star}$ into elongated distal rods that are incapable of puncturing the outer membrane. Yet, despite these deficiencies, the assembly program continues unabated and succeeds in forming the hook and the flagellar filament structures in the periplasm. Toward elucidating the molecular basis of these stop-polymerization effects, the FlgG ${ }^{\star}$ mutations were modeled onto the structure of the FlgE hook protein, which exhibits a high degree of sequence similarity to FlgG (Liu and Ochman 2007). This comparison indicated that most of the $\mathrm{FlgG}^{\star}$ mutations fall into exposed regions (one at the top and one at the bottom of the FlgE structural model), where two symmetrical FlgG-FlgG interfaces could interact. Chevance et al. (2007) speculate that the homomeric FlgG interaction would normally impose a conformational constraint that prevents the incorporation of additional FlgG molecules, thus limiting rod elongation by a "stop-polymerization" mechanism (Fig. 1A).

\section{Trans-membrane organelles: structures that push the envelope}

The stop-polymerization hypothesis offers an attractive explanation for the remarkable structural consistency of the flagellum and its ability to coordinate assembly events such as rod elongation with outer membrane penetration. The structural abnormalities observed in FlgG * mutants might hold the key to other, yet poorly understood events of flagellum biogenesis. For example, the observation that additional, but irregularly placed P-rings are found on elongated $\mathrm{FlgG}^{\star}$ rods is tantalizing. Runaway rod polymerization could increase the number of available nucleation sites, setting the stage for ectopic P-ring deposition. The observed irregularity might simply be a consequence of limiting the number of P-ring subunits relative to the rod (Chevance et al. 2007) that would stochastically assemble around nucleation sites that are in abundance. Such a hypothetical imbalance of P-ring to $\mathrm{FlgG}^{\star}$ subunits is conceivable, as the latter are transported into the periplasm through the Sec-dependent secretion pathway (Macnab 1999), which might be kinetically more restrained than the flagellar secretion machinery responsible for the export of the former. Alternatively, it is possible that the stability of these subunits is affected by the FlgG ${ }^{\star}$ mutation, resulting in an imbalance of the two proteins. Finally, the irregularities in the rotational staggering of $\mathrm{FlgG}^{\star}$ subunits might affect the effectiveness of P-ring nucleation or their number, thus forming P-ring distribution patterns that appear random. The absence of L-rings is also intriguing, as their formation could hinge on exact arrangements of P-rings or specialized sites not presented by elongated $\mathrm{FlgG}^{\star}$ rods. An attractive idea put forth by Chevance et al. (2007) to account for the lack of L-rings is that FlgG ${ }^{\star}$ mutations might result in temporal uncoupling of rod assembly from L-ring formation. If true, elongating $\mathrm{FlgG}^{\star}$ rods could encounter the outer membrane barrier before L-rings have formed, which might force the elongating rods into a bend. As a consequence, the rods would adopt a conformation that is parallel rather than perpendicular to the cell membrane, which would place the tip of the rod in the wrong orientation to coordinate L-ring formation (Fig. 1D; Chevance et al. 2007).

Another interesting question that remains to be addressed is whether the elongated $\mathrm{FlgG}^{\star}$ rods in the periplasm traverse the peptidoglycan layer with reduced efficiency. Upon formation of the distal rod in wild-type cells, the secretion machinery exports FlgJ, a muramidase that aides the penetration process by hydrolyzing peptidoglycan (Nambu et al. 1999). The FlgJ $\mathrm{N}$ terminus caps the nascent rod, while the C-terminal part forms a pore in the peptidoglycan, its substrate (Hirano et al. 2001). In the runaway polymerization context, rod elongation might impair transport (secretion substrate specificity) or delivery of FlgJ to the appropriate locale. Moreover, misalignment of the rod could direct FlgJ away from the peptidoglycan layer, or elongated rod structures could fail to bind the FlgJ capping domain, causing diffusion of the hydrolase into the periplasm. Experiments focused on the localization of FlgJ and its activity in $\mathrm{FlgG}^{\star}$ mutants will undoubtedly reveal whether any of these hypotheses ring true.

The notion of conformational coupling in trans-envelope substructures to elicit changes in secretion specificity is not unprecedented. Structural modeling of another 
trans-envelope protein complex in Salmonella, the Type III secretion system (T3SS)-based injectisome, also led to a model in which a conformational change brought about by the completion of the inner rod triggers a switch in substrate secretion specificity (Galan and Wolf-Watz 2006). The conformational deadlock caused by mutations that impair rod formation/completion prevent substrate switching and ultimately results in aberrant polymerization of the subunits that are continuously secreted (Marlovits et al. 2006). The parallel between these two observations should not go unnoticed considering the evolutionary kinship of these structures and their mechanisms of substrate switching. The assembly of other envelope-spanning complexes, such as unrelated protein secretion or metabolite extrusion/uptake systems, might also require strict elongation control of specific substructures. In analogy to the flagellar assembly process, these mechanisms might serve to coordinate polymerization of substructures with penetration of outer envelope layers to prevent the formation of aberrant polymers in the periplasm. It is noteworthy in this regard that certain combinations of mutations in the Type II-based pilus secretion system in Neisseria gonorrhoeae give rise to pilus filaments localized to the periplasm rather than the cell surface (Wolfgang et al. 2000). Whether a comparable stop-polymerization mechanism also underlies proper control of this filament or that of another substructure remains to be determined.

\section{Understanding organelle evolution: the writing is on the structure}

The stop-polymerization concept for the rod can also be extended to the formation of protein complexes in the cytoplasm (Briegel et al. 2006). The flagellar switch protein FliM (Fig. 1B) polymerizes into the cytoplasmic Cring, in which subunits are ordered side-by-side (Park et al. 2006). FliM subunit interactions impose a uniform conformational state that only changes cooperatively upon interactions with the structurally related CheY protein. In their model, Park et al. (2006) suggest that interactions between a CheY domain that resembles FliM and a single FliM ring constituent provides the signal that induces conformational switching. In the FlgG context, it is conceivable that stop-polymerization-like signals, exhorted by interdomain interactions, are at play here as well. In addition to the flagellum, stop-polymerization mechanisms can be applied to explain properties of other unrelated protein assemblages.

The actin-like MreB protein is thought of as a principal agent of cellular organization (Carballido-Lopez 2006). MreB monomers can self-associate to form filaments and reorganize into assemblies with specialized properties. MreB polymerizes into filamentous bundles through polarized end-to-end addition (Esue et al. 2005; Kim et al. 2006). Shearing of filamentous bundles to create new ends does not lead to renewed polymerization at these sites in vitro, pointing to specific polymerization requirements that are likely to have a structural basis. In addition to self-assembly, there appears to be an intrinsic ability of MreB to regulate its own multimeric conformational states (Esue et al. 2005). MreB filaments form spirals in vivo that can "morph" into discrete rings before cell division, a process that can be reconstituted in vitro without the addition of any other factors. Thus, MreB polymers exist in a number of different conformations that appear to answer to a "thermodynamic switch," establishing the environment for monomer addition and macromolecular switching. Other intracellular polymers built from tubulin or intermediate filament-like subunits also appear to be under strict elongation control, perhaps through functionally analogous concepts (Ausmees et al. 2003; Michie and Lowe 2006; Erickson 2007; Larsen et al. 2007). Thus, it is conceivable that different stop-polymerization mechanisms act on distinct conformational states of a given protein to modulate the overall structure and, thus, impart specialized function on such protein assemblages. Conservation of signaling events based on mechanical effects in seemingly diverse proteins could be extremely relevant considering the recent unveiling of evolutionary relationships between members of the core flagellum gene repertoire (Liu and Ochman 2007). Meta-genome and phylogenetic analyses indicate a gradual invention, starting from a single ancestral gene that duplicated and diversified (Liu and Ochman 2007). Addition of novel components allowed extension of the structure outward, eventually resulting in the extracellular flagellum. If stop-polymerization mechanisms arose during this process, it could be conserved and thus applicable to other protein assemblage events and related structures. The observation of a periplasmic flagellum in Salmonella is exciting and certainly provides sufficient grounds for provocative hypotheses. One idea put forward, for instance, is that mutation of FlgG recreates an evolutionary event that changed the orientation of the flagellum outward, suggesting that this represents a real event in flagellum evolution. Whether true or not, the fact that structural information is available that explains stoppolymerization and that this hypothesis can be tested directly will significantly advance our understanding of flagellum biogenesis.

\section{Conclusions}

In an age of high throughput, high resolution, high sensitivity, and ever-expanding databases, recognizing conceptual leaps and planting such advances into context can be a challenge. The study by Chevance et al. (2007) likely presents a key conceptual advance in the field of organelle biology and the stop-polymerization model fills a void in the poorly defined realm of complex protein assembly pathways. Furthermore, their findings may provide support for current evolutionary models that invoke a gradual invention of the flagellum (Chevance et al. 2007). Rational interpretation of their data would endorse a baby-step evolution and assembly mechanism for the flagellum, adding yet another silver bullet to the current "intelligent design/irreducible complexity" fallacy. 


\section{Acknowledgments}

Research in P.V.'s laboratory is supported by funds from the School of Medicine, the Mount Sinai Health Care Foundation, and the US Department of Energy Office of Science (BER, grant no. DE-FG02-05ER64136).

\section{References}

Aldridge, P. and Hughes, K.T. 2002. Regulation of flagellar assembly. Curr. Opin. Microbiol. 5: 160-165.

Aldridge, P., Karlinsey, J.E., Becker, E., Chevance, F.F., and Hughes, K.T. 2006. Flk prevents premature secretion of the anti- $\sigma$ factor FlgM into the periplasm. Mol. Microbiol. 60: 630-643.

Ausmees, N., Kuhn, J.R., and Jacobs-Wagner, C. 2003. The bacterial cytoskeleton: An intermediate filament-like function in cell shape. Cell 115: 705-713.

Bardy, S.L., Ng, S.Y., and Jarrell, K.F. 2003. Prokaryotic motility structures. Microbiology 149: 295-304.

Briegel, A., Dias, D.P., Li, Z., Jensen, R.B., Frangakis, A.S., and Jensen, G.J. 2006. Multiple large filament bundles observed in Caulobacter crescentus by electron cryotomography. Mol. Microbiol. 62: 5-14.

Carballido-Lopez, R. 2006. The bacterial actin-like cytoskeleton. Microbiol. Mol. Biol. Rev. 70: 888-909.

Chevance, F.F.V., Takahashi, N., Karlinsey, J.E., Gnerer, J., Hirano, T., Samudrula, R., Aizawa, S.-I., and Hughes, K.T. 2007. The mechanism of outer membrane penetration by the eubacterial flagellum and implications for spirochete evolution. Genes \& Dev. (this issue), doi: 10.1101/gad.1571607.

Erickson, H.P. 2007. Evolution of the cytoskeleton. Bioessays 29: 668-677.

Esue, O., Cordero, M., Wirtz, D., and Tseng, Y. 2005. The assembly of MreB, a prokaryotic homolog of actin. J. Biol. Chem. 280: 2628-2635.

Galan, J.E. and Wolf-Watz, H. 2006. Protein delivery into eukaryotic cells by type III secretion machines. Nature 444: 567-573.

Gillen, K.L. and Hughes, K.T. 1991. Molecular characterization of $f l g M$, a gene encoding a negative regulator of flagellin synthesis in Salmonella typhimurium. I. Bacteriol. 173: 6453-6459.

Hirano, T., Minamino, T., and Macnab, R.M. 2001. The role in flagellar rod assembly of the N-terminal domain of Salmonella FlgJ, a flagellum-specific muramidase. J. Mol. Biol. 312: 359-369.

Hughes, K.T., Gillen, K.L., Semon, M.J., and Karlinsey, J.E. 1993. Sensing structural intermediates in bacterial flagellar assembly by export of a negative regulator. Science 262: 1277-1280.

Huitema, E., Pritchard, S., Matteson, D., Radhakrishnan, S.K., and Viollier, P.H. 2006. Bacterial birth scar proteins mark future flagellum assembly site. Cell 124: 1025-1037.

Karlinsey, J.E., Tanaka, S., Bettenworth, V., Yamaguchi, S., Boos, W., Aizawa, S.I., and Hughes, K.T. 2000. Completion of the hook-basal body complex of the Salmonella typhimurium flagellum is coupled to FlgM secretion and fliC transcription. Mol. Microbiol. 37: 1220-1231.

Kim, S.Y., Gitai, Z., Kinkhabwala, A., Shapiro, L., and Moerner, W.E. 2006. Single molecules of the bacterial actin MreB undergo directed treadmilling motion in Caulobacter crescentus. Proc. Natl. Acad. Sci. 103: 10929-10934.

Lam, H., Schofield, W.B., and Jacobs-Wagner, C. 2006. A landmark protein essential for establishing and perpetuating the polarity of a bacterial cell. Cell 124: 1011-1023.
Larsen, R.A., Cusumano, C., Fujioka, A., Lim-Fong, G., Patterson, P., and Pogliano, J. 2007. Treadmilling of a prokaryotic tubulin-like protein, TubZ, required for plasmid stability in Bacillus thuringiensis. Genes \& Dev. 21: 1340-1352.

Liu, R. and Ochman, H. 2007. Stepwise formation of the bacterial flagellar system. Proc. Nat1. Acad. Sci. 104: 7116-7121.

Macnab, R.M. 1999. The bacterial flagellum: Reversible rotary propeller and type III export apparatus. J. Bacteriol. 181: 7149-7153.

Macnab, R.M. 2003. How bacteria assemble flagella. Annu. Rev. Microbiol. 57: 77-100.

Marlovits, T.C., Kubori, T., Lara-Tejero, M., Thomas, D., Unger, V.M., and Galan, J.E. 2006. Assembly of the inner rod determines needle length in the type III secretion injectisome. Nature 441: 637-640.

Michie, K.A. and Lowe, J. 2006. Dynamic filaments of the bacterial cytoskeleton. Annu. Rev. Biochem. 75: 467-492.

Minamino, T. and Macnab, R.M. 1999. Components of the Salmonella flagellar export apparatus and classification of export substrates. J. Bacteriol. 181: 1388-1394.

Minamino, T., Doi, H., and Kutsukake, K. 1999. Substrate specificity switching of the flagellum-specific export apparatus during flagellar morphogenesis in Salmonella typhimurium. Biosci. Biotechnol. Biochem. 63: 1301-1303.

Murphy, G.E., Leadbetter, J.R., and Jensen, G.J. 2006. In situ structure of the complete Treponema primitia flagellar motor. Nature 442: 1062-1064.

Murray, T.S. and Kazmierczak, B.I. 2006. FlhF is required for swimming and swarming in Pseudomonas aeruginosa. J. Bacteriol. 188: 6995-7004.

Nambu, T., Minamino, T., Macnab, R.M., and Kutsukake, K. 1999. Peptidoglycan-hydrolyzing activity of the FlgJ protein, essential for flagellar rod formation in Salmonella typhimurium. J. Bacteriol. 181: 1555-1561.

Pandza, S., Baetens, M., Park, C.H., Au, T., Keyhan, M., and Matin, A. 2000. The G-protein FlhF has a role in polar flagellar placement and general stress response induction in Pseudomonas putida. Mol. Microbiol. 36: 414-423.

Park, S.Y., Lowder, B., Bilwes, A.M., Blair, D.F., and Crane, B.R. 2006. Structure of FliM provides insight into assembly of the switch complex in the bacterial flagella motor. Proc. Natl. Acad. Sci. 103: 11886-11891.

Wolfgang, M., van Putten, J.P., Hayes, S.F., Dorward, D., and Koomey, M. 2000. Components and dynamics of fiber formation define a ubiquitous biogenesis pathway for bacterial pili. EMBO J. 19: 6408-6418. 


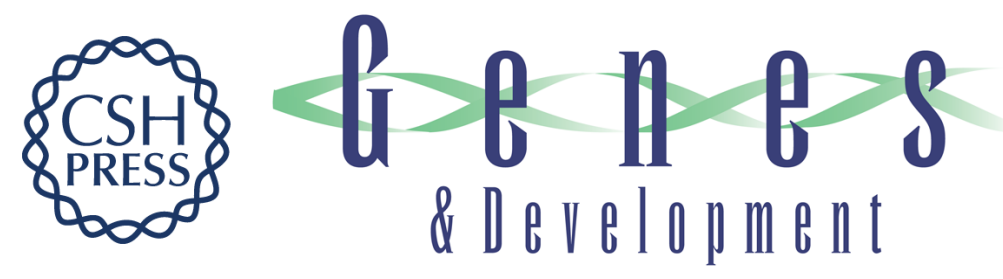

\section{Break on through to the other side: outer membrane penetration of the nascent flagellum by a stop-polymerization mechanism}

Edgar Huitema and Patrick H. Viollier

Genes Dev. 2007, 21:

Access the most recent version at doi:10.1101/gad.1600807
Related Content The mechanism of outer membrane penetration by the eubacterial flagellum and implications for spirochete evolution
Fabienne F.V. Chevance, Noriko Takahashi, Joyce E. Karlinsey, et al.
Genes Dev. September, 2007 21:2326-2335
References This article cites 30 articles, 13 of which can be accessed free at:
http://genesdev.cshlp.org/content/21/18/2253.full.html\#ref-list-1
Articles cited in:
http://genesdev.cshlp.org/content/21/18/2253.full.html\#related-urls

\section{License}
Email Alerting
Service
Receive free email alerts when new articles cite this article - sign up in the box at the top right corner of the article or click here.

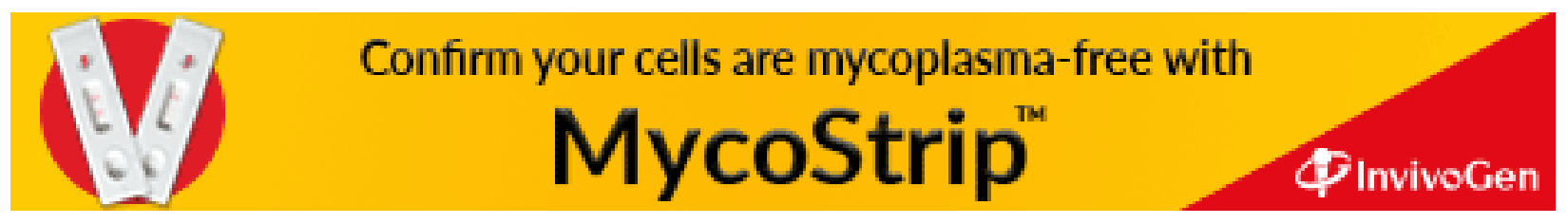

